\title{
Early termination of cognitive-behavioural interventions: literature review
}

\author{
Alberto Salmoiraghi, ${ }^{1,2}$ Rajvinder Sambhi ${ }^{1}$
}

The Psychiatrist (2010), 34, 529-532, doi: 10.1192/pb.bp.110.030775

${ }^{1}$ Betsi Cadwaladr University Health Board, Wrexham; ${ }^{2}$ University of Liverpool

Correspondence to Alberto

Salmoiraghi

(alberto.salmoiraghi@wales.nhs.uk)

\begin{abstract}
Aims and method The evidence of the efficacy of cognitive-behavioural interventions is well established. Despite this, clinicians often face a consistent number of not attended appointments or early treatment discontinuation rates. This paper reviews the literature to date regarding the possible causes of early termination of cognitive-behavioural interventions. A literature search was done using Medline, PsycINFO, Embase and the Cochrane Library.
\end{abstract}

Results We reviewed 14 articles. Out of these, two were randomised controlled trials, one was a controlled trial and the rest were cohort studies. Drop-out was defined differently across papers. There are many factors that may affect early treatment discontinuation rates and they are described individually.

Clinical implications There does not appear to be a single strong predictor of early treatment discontinuation. Specific literature relating to cognitive-behavioural therapy is scarce. However, some factors such as young age, education level and psychopathology appear to be more strongly associated with early treatment discontinuation than others.

Declaration of interest None.
Cognitive-behavioural therapy (CBT) is a well-recognised psychotherapeutic intervention for the treatment of a number of psychiatric conditions such as depression, anxiety, eating disorders and obsessive-compulsive disorder. ${ }^{1}$ Furthermore, CBT techniques have proven to be efficacious in targeting specific conditions such as chronic delusions and hallucinations in schizophrenia. Lord Layard examined the need for psychological treatments in the UK and speculated that there will be a demand to train 5000 more psychological therapists by 2013 , mostly in CBT. ${ }^{2}$

However, clinicians practising CBT note a consistent number of cancelled or not attended appointments. More importantly, a number of patients start the therapeutic process but terminate the therapy earlier than agreed, without comprehensively explaining the reasons or demonstrating a consolidation of the results. This is despite the fact that some patients appear initially to be well engaged or even show a marked improvement with a relatively low number of sessions.

Attrition rates in psychotherapy are high, ranging from 20 to $60 \%$ depending on the setting where the intervention is delivered and the modality of the intervention. ${ }^{3-11}$ The difficulty in establishing a precise figure lies in the fact that there is no uniformity in the definition of treatment discontinuation. ${ }^{3}$ Some researchers define early treatment discontinuation as terminating the treatment without previous agreement with the therapist, others use the number of visits or the length of time as criteria. The definition affects the results, but consensus has not been established. ${ }^{12}$

Treatment discontinuation represents a significant problem in mental health, as it seems that generally the outcomes of those who dropped out of treatment are poorer than for people who stay in treatment or are equivalent to outcomes of those who were untreated, ${ }^{13-5}$ and $21-46 \%$ of those patients end up getting treated in another setting within the next year., ${ }^{3,4}$ Furthermore, treatment discontinuation constitutes a financial loss for services in taxpayer-funded systems such as the National Health Service (NHS) in the UK and for the individual patients themselves. $^{8}$ This may reduce the therapist's job satisfaction, which in turn may lead to increased staff turnover. ${ }^{14}$ The same author argues on an emotional cost to the therapists. ${ }^{14}$ With regard to the economic aspect, some researchers ${ }^{16-8}$ argue that treatment discontinuation represents a waste of resources in a system where the demand for psychotherapy exceeds the limited supply. Davis et $a l^{9}$ point out that the overall efficacy of CBT interventions is lessened when treatment discontinuation is considered.

We are of the opinion that addressing the problem of early treatment discontinuation is paramount in view of optimising any publicly funded healthcare system, the allocation of resources and the waiting lists. In this paper we review the literature specifically on attrition in highintensity CBT interventions. 


\section{Method}

An electronic literature search was done by using Medline, PsycINFO, Embase and the Cochrane Library. Key terms used were 'CBT', 'cognitive behaviour therapy/cognitive therapy', 'patient dropouts', 'early termination', 'patient compliance' and 'psychotherapy'. The terms 'CBT', 'psychotherapy' and 'cognitive therapy/cognitive behaviour therapy' were initially combined to get the results; the same was done for 'early termination', 'patient dropouts' and 'patient compliance'. After this, the results of both the combinations were combined to get the final numbers. Duplicates were excluded. We did not set any restriction regarding the language or the year of publication. We did not search for unpublished articles. We screened all the abstracts to select the relevant articles, including all the papers that explicitly mentioned 'dropout' and CBT interventions either in the title or in the abstract. We have excluded articles that specifically described non-CBT psychotherapies, outcomes other than early treatment termination and all the articles that mentioned the general term 'psychotherapy'. All types of studies were considered, including those of lower quality or opinion papers. Every article selected was reviewed by both A.S. and R.S., including references for additional cited articles. Finally, the search was completed by cross-referencing the selected papers.

\section{Results}

In Medline, Embase and PsycINFO, the keyword 'early termination' generated 1625 results, 'CBT' 9574, 'psychotherapy' 69115 and 'cognitive therapy/cognitive behaviour therapy' 21468 results. Combining the terms 'CBT', 'psychotherapy' and 'cognitive therapy/or cognitive behaviour therapy' generated 94666 results. The term 'patient dropouts' generated 4597 results and 'patient compliance' 21796 results; the combination of these two terms generated 27827 results. The combination of all the search terms generated a total of 582 abstracts. After removing the duplicates, we obtained 516 abstracts. Finally, 179 abstracts were selected for the initial screening, based on the inference from the title as to whether the abstract would be dealing with CBT or 'dropouts'. The final screening and review of these 179 abstracts generated 14 articles that fulfilled the criteria for inclusion in this review. Two of these articles were randomised controlled trials, one was a controlled trial and the rest were cohort studies, both prospective and retrospective (Fig. 1).

There is some controversy in the results regarding the relationship between demographic variables and patients who drop out of treatment. In general, it seems that younger service users with low education level and of lower social class background are more likely to drop out of CBT., ${ }^{4,91}$ In a randomised controlled trial by Arnow et $a l,{ }^{11}$ those of ethnic minority background are reported to have significantly higher drop-out rates. However, in a cohort study of group CBT for depression, ${ }^{7}$ age and education level did not statistically differ between those who dropped out, whether early or late, and those who completed the therapy. Similar results are reported in other studies where general
Electronic search (Medline, Embase, PsychINFO) for early treatment discontinuation in CBT using specific key words

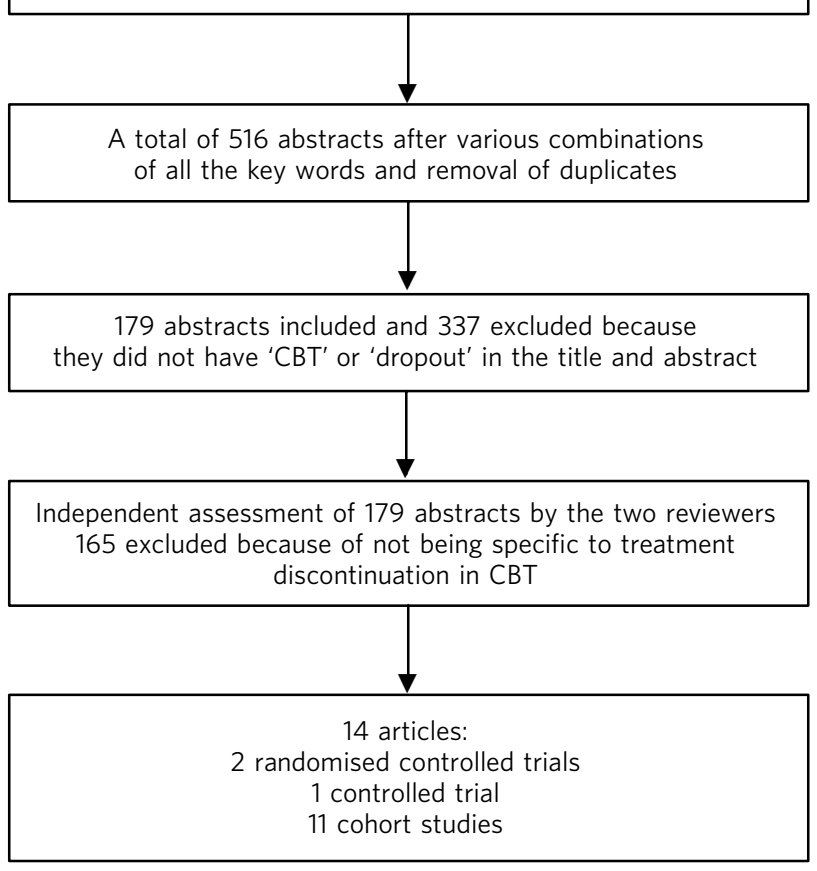

Fig 1 Study process.

CBT, cognitive-behavioural therapy.

demographic variables did not differ between the two groups. ${ }^{19-23}$

The diagnosis is another contentious issue. There is no robust evidence supporting an association between any particular diagnosis and early treatment discontinuation rates, ${ }^{12}$ a finding confirmed by all the studies in that review. However, Persons et $a l^{4}$ report that patients with personality disorders were more likely to drop out of treatment. In a study of early discontinuation of CBT for bulimia, ${ }^{23}$ higher level of borderline and dissociative symptomatology was associated with discontinuation. These findings are not supported by Arnow et al's study, ${ }^{11}$ in which comorbid personality disorders were equally prevalent in those who completed the treatment and those who dropped out, and only anxiety disorder correlated to the latter. The severity of the depressive symptomatology seems to be correlated with early discontinuation rates. ${ }^{4,22,25}$

Common sense suggests that practicalities may play a role in a number of patients who do not return to therapy. These include difficulties with transport, finances, busy timetables or time constraints, clash with other commitments, personal problems and taking time off work. Only two studies ${ }^{12,19}$ reported significant results for those variables, one citing high scores for time and transportation. ${ }^{19}$ Surprisingly, only one study reported dissatisfaction with treatment or therapist as a reason for terminating therapy. ${ }^{12}$

There are references to other causes of early treatment discontinuation in the literature, although sometimes they are only mentioned or partially explored. Davis et $a l^{9}$ examined data from an archive of group CBT and identified low self-esteem at admission and poor relationship status as 
the only two predictors of treatment discontinuation. However, in a controlled trial by the same authors, completing a self-esteem module before commencing group CBT did not decrease the drop-out rate. ${ }^{9}$ In a cohort study by Steel et $a l^{20}$ on predictors of early treatment termination in CBT for bulimia nervosa, the patients who were more likely to drop out had a higher weight fluctuation, scored higher in the subscale of Ineffectiveness of the Eating Disorder Inventory-2 (feelings of general inadequacy, insecurity, worthlessness, emptiness, low self-esteem and negative self-evaluation), and had a higher level of pre-treatment depression, hopelessness and external locus of control. Similar results are reported in a study by Coker et $a l^{22}$ In a study by Westra et $a l_{,}{ }^{24}$ low selfesteem and pre-treatment hopelessness were also predictors of drop-out in cognitive-behavioural group therapy for depression. Short total sleep time was reported to be associated with treatment discontinuation in a paper on CBT for insomnia. ${ }^{25}$ In another study of CBT in eating disorders in an in-patient setting investigating personality dimensions of those who discontinued their treatment $v$. those who completed it, Dalla Grave et $a l^{21}$ found only one significant difference in the subscale of Persistence in the Temperament and Character Inventory.

Finally, in a randomised trial, Arnow et $a l^{11}$ found a statistically significant difference in early working alliance total scores between those who completed their psychotherapy treatment and those who did not.

\section{Discussion}

From this review it emerged that there is little specific literature investigating a very common and expensive problem such as early treatment discontinuation in CBT interventions. Furthermore, some of the literature is about treatments for specialist conditions such as eating disorders and insomnia. We could not find any paper addressing treatment discontinuation in CBT for anxiety disorders and found only a few for depression. This review seems to indicate that there is no single robust predictor of treatment discontinuation in CBT. Another finding is that the prevalence of treatment discontinuation in CBT interventions ranges from 19 to $50 \%$ across studies.

The strength of this review is that the selection criteria were very strict, with the exclusion of every paper mentioning the general term psychotherapy. This was because psychotherapy includes a variety of interventions other than CBT. Findings about treatment discontinuation and general psychotherapy do not necessarily apply to CBT interventions. However, paradoxically this is also a limitation of the study, as this has undoubtedly excluded seemingly important findings about treatment discontinuation. Moreover, we reviewed only those articles in which the terms 'CBT' and 'dropouts' appeared either in the title or in the abstract. We recognise that this may have led to excluding a large number of papers mentioning 'dropouts' in different contexts in the main body.

Hunt \& Andrews ${ }^{26}$ suggested that high discontinuation rates are an indicator of poor performance in psychotherapy. This is arguably a very good point and perhaps services should invest more into decreasing those rates, for example through sponsoring research projects to establish which factors can be addressed and modified in the short and long term. The NHS information service in the UK calculated an average cost of a CBT session as $£ 58 .^{27}$ Even a small reduction in non-attendance rates will produce substantial savings. Furthermore, the optimisation of resources may have an effect on staff morale and the public image of health services.

There does not appear to be any consensus on specific factors responsible for treatment discontinuation in CBT interventions. More systematic research is needed to address this very common issue as this has a huge potential in terms of quality of services delivered and their economic value.

\section{About the authors}

Alberto Salmoiraghi, consultant psychiatrist and North Wales Head of Acute Care Programme, Betsi Cadwaladr University Health Board Wrexham, and Honorary Lecturer, School of Population, Community and Behavioural Sciences, Department of Psychiatry, University of Liverpool, Rajvinder Sambhi, specialty registrar in psychiatry, Betsi Cadwaladr University Health Board, Wrexham.

\section{References}

1 Department of Health. Treatment Choice in Psychological Therapies and Counselling: Evidence Based Clinical Practice Guideline. Department of Health, 2001.

2 Layard R. The case of psychological treatment centres. BMJ 2006; 332: 1030-2.

3 Baekeland F, Lundwall L. Dropping out of treatment: a critical review. Psychol Bull 1975; 82: 738-83.

4 Persons JB, Burns DD, Perloff JM. Predictors of dropout in cognitive therapy for depression in a private practice setting. Cogn Ther Res 1988; 12: $557-75$.

5 Pekarik G. Post treatment adjustment of clients who drop out early vs. late in treatment. J Clin Psychol 1992; 48: 379-87.

6 Wierzbicki M, Pekarik G. A meta-analysis of psychotherapy dropout. Prof Psychol Res Pr 1993; 24: 90-195.

7 Oei TPS, Kazmierczak T. Factors associated with dropout in a group cognitive behaviour therapy for mood disorders. Behav Res Ther 1997; 35: $1025-30$.

8 Brogan MM, Prochaska JO, Prochaska JM. Predicting termination and continuation status in psychotherapy using the transtheoretical model. Psychotherapy 1999; 36: 105-13.

9 Davis S, Hooke GR, Page AC. Identifying and targeting predictors of drop-out from group cognitive behaviour therapy. Aust J Psychol 2006; 58: $48-56$.

10 Principe JM, Marci CD, Glick DM, Ablon JS. The relationship among patient contemplation, early alliance and continuation in psychotherapy. Psychother Theory Res Pr Training 2006; 43: 238-43.

11 Arnow BA, Blasey C, Manber R, Constantino MJ, Markowitz JC, Klein $\mathrm{DN}$, et al. Dropouts versus completers among chronically depressed outpatients. J Affect Disord 2007; 97: 197-202.

12 Bados A, Balaguer G, Saldaña C. The efficacy of cognitive-behavioural therapy and the problem of dropout. J Clin Psychol 2007; 63: 585-92.

13 Pekarik G. Improvement in clients who have given different reasons for dropping out of treatment. J Clin Psychol 1983; 39: 909-13.

14 Pekarik G. Coping with dropouts. Prof Psychol Res Pr 1985; 16: 114-23.

15 Masi MV, Miller RB, Olson MM. Differences in dropout rates among individual, couple, and family therapy clients. Contemp Fam Ther 2003; 25: $63-75$. 
16 Hillis G, Alexander DA, Eagles J M. Premature termination of psychiatric contact. Int J Soc Psychiatry 1993; 39: 100-7.

17 Frank JD, Gliedman LH, Stanley DI, Nash Jr EH, Stone AR. Why patients leave psychotherapy. Arch Neurol Psychiatry 1957; 77: 283-99.

18 Borghi $\mathrm{JH}$. Premature termination of psychotherapy and patienttherapist expectations. Am J Psychother 1968; 22: 460-73.

19 Simons AD, Levine JL, Lustman PJ, Murphy GE. Patient attrition in a comparative outcome study of depression: a follow-up report. J Affect Disord 1984; 6: 163-73.

20 Steel Z, Jones J, Adcock S, Clancy R, Bridgford-West L, Austin J. Why the high rate of dropout from individualized cognitive-behaviour therapy for bulimia nervosa? Int J Eat Disord 2000; 28: 209-14.

21 Dalle Grave R, Calugi S, Brambilla F, Marchesini G. Personality dimensions and treatment drop-outs among eating disorder patients treated with cognitive behaviour therapy. Psychiatry Res 2008; 158: $381-8$
22 Coker S, Vize C, Wade T, Cooper PJ. Patients with bulimia nervosa who fail to engage in cognitive behaviour therapy. Int J Eat Disord 1993; 13: 35-40.

23 Waller G. Drop-out and failure to engage in individual outpatient cognitive behaviour therapy for bulimic disorders. Int J Eating Disord 1997; 22: 35-41.

24 Westra HA, Dozois DJA, Boardman C. Predictors of treatment change and engagement in cognitive-behavioural group therapy for depression. J Cogn Psychotherapy 2002; 16: 227-41.

25 Ong JC, Kuo TF, Manber R. Who is at risk for dropout from group cognitive-behaviour therapy for insomnia? J Psychosom Res 2008; 64 $419-25$.

26 Hunt C, Andrews G. Dropout rate as a performance indicator in psychotherapy. Acta Psychiatr Scand 1992; 85: 275-8.

27 Curtis L. Unit Costs of Health and Social Care. 2008. Personal Social Services Research Unit, The University of Kent, 2008 (http:// www.pssru.ac.uk/pdf/uc/uc2008/uc2008.pdf). 